\title{
La influencia de un modelo constructivista para la enseñanza de la táctica en baloncesto sobre la eficacia del juego durante la competición The influence of a constructivist model for teaching tactics in basketball on the effectiveness of the game during the competition
}

\author{
*Francisco Alarcón López, **David Cárdenas Vélez, **M. Teresa Miranda León, ***Nuria Ureña Ortín, **M. Isabel Piñar López \\ *Universidad Católica San Antonio de Murcia, **Universidad de Granada, ***Universidad de Murcia
}

Resumen: El rendimiento en baloncesto viene determinado no sólo por la capacidad de ejecución de los participantes sino por la capacidad de superar al adversario que genera un entorno cambiante, donde el jugador debe estar analizando y decidiendo continuamente las acciones a realizar para conseguir encestar. El objetivo de este estudio fue analizar si con un programa de entrenamiento para la táctica, basado en un modelo constructivista, se puede conseguir mejorar el rendimiento de un equipo de baloncesto masculino formado por 10 jugadores seniors. Para ello se utilizaron como variables dependientes la eficacia de los ataques posicionales y la eficacia de los medios tácticos, entendiendo ésta como la capacidad de conseguir ventajas espaciales del jugador con balón. La recogida de datos se realizó mediante metodología observacional gracias a un instrumento previamente validado. Se utilizó un diseño pretest-postest sin grupo control. Los resultados mostraron que existió una mejora en la eficacia de los ataques posicicionales con diferencias muy significativas, pasando del $28,6 \%$ de eficacia en el pretest al $67,5 \%$ en el postest. Estos mismos resultados se repitieron con la eficacia de los medios tácticos, esta vez con diferencias altamente significativas, pasando de una eficacia del $29,5 \%$ en el pretest a una del $64,9 \%$ en el postest. La principal conclusión del estudio es que un programa de entrenamiento para la enseñanza de la táctica colectiva basado en un modelo constructivista tiene una influencia positiva en el rendimiento de un equipo de baloncesto.

Palabra clave: táctica, enseñanza, constructivismo, eficacia.

\begin{abstract}
The performance in basketball comes determined not only for the capacity of execution of the participants but for the aptitude to overcome the adversary who generates a changeable environment, where the player must be analyzing and deciding constant the actions to obtain to score. The aim of this study was to analyze if with a program of training for the tactics, based on a model constructivism, it is possible to manage to improve the performance of a male basketball team formed by 10 players seniors. For it there were in use as dependent variables the efficacy of positional attacks and the effectiveness of tactical media, understood as the ability to achieve benefits of space with ball player.. The data Collection of information was realized by means of methodology observational thanks to a before validated instrument. A design was in use pretest-postest without group control. The results showed that an improvement existed in the efficiency of the assaults posicicionales with very significant differences, happening from $28,6 \%$ of efficiency in the pretest to $67,5 \%$ in the postest.The same results repeated themselves with the efficiency of the tactical means, this time with highly significant differences, happening from an efficiency of $29,5 \%$ in the pretest to one from $64,9 \%$ in the postest. The principal conclusion of the study is that a program of training for the education of the collective tactics based on a model constructivist has a positive influence in the performance of a basketball team.
\end{abstract}

Key words: tactics, education, constructivism, efficiency.

\section{Introducción}

Según el reglamento de los deportes de equipo vence el equipo que consiga un mayor número de puntos en el tiempo establecido. En baloncesto la forma de conseguir anotar es introducir el balón en el aro del equipo rival, lo que convierte dicha acción en la más importante y el objetivo prioritario. Por este motivo cuando se ha querido analizar las variables que inciden en el rendimiento de los equipos, muchos autores han utilizado el porcentaje de acierto en los tiros de campo (Fierro, 2002; Gómez y Lorenzo, 2005; Karipidis, Fotinakis, Taxildaris, Filaktakidou y Fatouros, 2001; Sampaio, 2000; Satter, 2003; Sampaio y Leite, 2006; Tsamourtzis y Athanasiou, N., 2004; Vidal y Mercadé, 2002), encontrando que los equipos ganadores tienen mejores porcentajes de tiros tanto de dos como de tres. También se han utilizado otras variables como las faltas recibidas (Amorim, 2001; Fierro, 2002; Manhao, 2000; Sampaio, 1998; Tsamourtzis, Fylaktakidou \& Taxildaris, 2001). Pero, a diferencia de los deportes individuales que normalmente necesitan dominar un solo gesto para conseguir su objetivo, en baloncesto se necesita más de una acción motriz específica para conseguir el objetivo de juego: encestar (Cárdenas, 2005). Estas acciones van encaminadas a conseguir que el lanzamiento se realice en las mejores condiciones posibles (Cárdenas, 1999).

Fecha recepción: 17-05-09 - Fecha envío revisores: 25-05-09 - Fecha de aceptación: 20-08-09 Correspondencia: Francisco Alarcón López

Universidad Católica San Antonio de Murcia.

Campus de los Jerónimos s/n. Guadalupe. 30107. Murcia

e-mail: falarcon@pdi.ucam.edu
Por lo que si queremos evaluar la eficacia de un equipo no sólo tendremos que hacerlo analizando la habilidad última, según los porcentajes de acierto en el lanzamiento, sino evaluando si el resto de habilidades han conseguido su objetivo, es decir, cumplir con unas condiciones óptimas que garanticen un buen lanzamiento, para lo cual los jugadores deben intentar superar al contrario momentáneamente para conseguir lanzar con el menor grado de oposición (Alarcón, 2008). Para ello es necesario dificultar la acción defensiva todo lo que se pueda y así conseguir ventajas espaciales con respecto los oponentes más cercanos. Estas ventajas suelen durar poco, puesto que, o el defensor recupera su posición perdida, o aparecen ayudas que neutralizan la ventaja conseguida. Una vez que el jugador ha conseguido una ventaja espacial durante el juego debería aprovecharla para conseguir encestar, ya sea lanzando sin oposición o aproximándose al aro y lanzando en sus cercanías donde los porcentajes de acierto son mayores. Aún consiguiendo lanzar en estas condiciones, los jugadores suelen errar muchos de estos lanzamientos, sobre todo por motivos puramente técnicos o psicológicos, no consiguiendo éxito. Pero, en estos casos, las decisiones que se tomaron llevaron a poder realizar un buen lanzamiento, por lo que desde un punto de vista táctico, el ataque fue eficaz. Según Refoyo (2001), la táctica colectiva, en el que se enfrentan a varios individuos, implica que éstos deben analizar la situación de los adversarios, oponentes y compañeros para decidir y ejecutar la acción colectiva más conveniente para la consecución del objetivo.

Por lo tanto se podría decir que la eficacia del juego colectivo no viene dada sólo por la conseguir encestar, sino por la consecución de ventajas espaciales durante las acciones colectivas.

Según lo expuesto un método de entrenamiento que se base en la mejora táctica debería aumentar las ventajas que se producen durante el juego. Además, al mejorar las condiciones en las que se produce el 
lanzamiento, debería también mejorar los porcentajes de tiro de campo, aún sin incidir en la mejora de la técnica del lanzamiento.

En la actualidad, y desde una corriente de aprendizaje cognitivista, existen muchos modelos de enseñanza que intentan adaptarse a las necesidades de la táctica en los deportes de equipo. Uno de éstos es el modelo constructivista, que se basa en las teorías de aprendizaje que dan nombre al modelo, las cuales hacen hincapié en la realización de aprendizajes con significado (Nuviala y Tamayo, 2003), es decir, que el jugador comprenda, dándole sentido al nuevo aprendizaje. Para ello, los jugadores deben tomar conciencia de la necesidad de los aprendizajes para afrontar los problemas que surgen en el juego (Butler, 1996). Además, las actividades que se propongan para este aprendizaje deben mantener el marco contextual y problemático que se da en el juego real (Grehaigne, Godbout, y Bouthier, 1999). Por último, los aprendices se deben involucrar activamente en el aprendizaje, tratando de relacionar lo que aprenden con lo que ya saben, siendo éstos «constructores» de sus aprendizajes, quedando la función del entrenador como mediador entre el nuevo conocimiento y el aprendiz (Contreras, 1998; Ureña, Alarcón y Ureña, 2009). A diferencia de otros modelos cognitivos, el modelo constructivista no se limita a lautilización exclusiva de juegos modificados, sino que incluye todo tipo de tareas que contengan elementos característicos de las prácticas deportivas estandarizadas, en el que se trata de mejorar las capacidades estratégicas y de ejecución de los participantes (Grehaigne, Godbout y Bouthier, 2001; Musch et al., 2002; González, Gutiérrez, Pastor, Fernández, 2007). Esto significa que este modelo no rechaza el aprendizaje de los aspectos técnicos, ni de la enseñanza repetitiva y analítica para conseguir la automatización de estas técnicas, sino que éstas se subordinan a las necesidades de que el jugador conozca y comprenda su significado en el juego, antes de automatizarlas (Alarcón, 2008).

Fundamentándose en esta forma de entender la intervención del entrenador, Cárdenas (1999) realiza una propuesta para los deportes colectivos, y que es la que se utiliza en este estudio para la mejora de la táctica colectiva en baloncesto. Existen ya algunos estudios que se basan en este planteamiento de la enseñanza constructivista (Harvey, 2003; Harvey, Bryan, Weigs, González \& Van Der Mars, 2006; Iglesias, 2006; Tallir, Lenoir, Valcke \& Musch, 2005; Wright, Mcneill, Fry \& Wang, 2005) y en los que se han encontrado mejoras sobre aspectos de la táctica como la toma de decisiones en deportes de equipo, aunque ninguno de ellos analizó si con esta metodología se podría conseguir mejorar la eficacia del juego durante la competición.

Según algunos autores cuando se realiza un proceso de enseñazaaprendizaje durante la competición ésta se convierte en una medida interesante para el propio estudio (McMorris y Graydon, 1997; Ponce, 2006). El objetivo por tanto de esta investigación será comprobar cómo varía la eficacia del juego colectivo posicional de un equipo de baloncesto formado por los sujetos participantes en el estudio, durante y tras la realización de un programa de intervención para la mejora de la táctica colectiva de ataque.

\section{Método}

\subsection{Muestra}

La población a la que se dirigió esta investigación estuvo compuesta por jugadores de baloncesto del Municipio de Murcia. Los sujetos que formaron parte de este estudio fueron los jugadores pertenecientes al equipo de la Universidad Católica de Murcia que militó en la $1^{\mathrm{a}}$ División Autonómica de la Región de Murcia. Este equipo constaba de 10 jugadores con edades comprendidas entre 18 y 26 años, con una media de edad de 21 años. El tipo de muestreo seleccionado fue un muestreo no probabilístico de carácter opinático(Sierra-Bravo, 1996) ó deliberado, cuya característica fundamental es que los sujetos fueron seleccionados de manera intencionada (Buendía, Colás y Hernández, 1998).

\subsection{Diseño y variables objeto de estudio de la investigación}

El presente trabajo de investigación utilizó un diseño cuasiexperimental antes-después (pretest-postest) sin grupo control, en el que el grupo considerado es el grupo experimental (Latorre, Del Rincón y Arnal, 2003). El pretest tuvo lugar durante la pretemporada con un rival de la misma categoría. Se eligió como postest el último partido de la fase de ascenso de la competición. También se seleccionó un partido por mesociclo al azar (un total de 8 partidos) para analizar la progresión de las variables dependientes.

En relación a las variables del estudio se registraron las siguientes variables dependientes:

Eficacia de los ataques posicionales. Las categorías fueron:

1. Ataque no eficaz. Aquel en el que no se consigue ningún beneficio ni en puntos, ni en faltas personales realizadas por el equipo contrario. Se considera ataque posicional la fase del juego que discurre a partir del transporte del balón a pista delantera, una vez que la defensa rival se encuentra correctamente posicionada para evitar la aproximación cómoda y rápida tanto del balón como de los jugadores hacia la canasta (Piñar, 2005), y en la que no existe superioridad numérica o táctica.

2. Ataque eficaz. Cuando se consigue puntuar por canasta convertida de dos o tres puntos, o por falta personal, con o sin tiro libre convertidos.

Eficacia de los medios tácticos colectivos básicos (MTCB $)$.

Esta eficacia vendrá dada por la obtención de la posesión de balón $\mathrm{y}$ ventaja por parte del beneficiario principal o secundario del MTCB. Así las categorías fueron:

1. Mediono eficaz: los beneficiarios no consiguen ventaja suficiente para obtener la posesión del balón.

2. Medio eficaz: uno de los participantes en el medio obtiene la posesión del balón o ventaja con el balón. Se define ventaja como el desequilibrio creado al adversario que defiende al jugador con balón, durante la realización del medio (puede ser cualquiera de los oponentes que participen en el medio) y que le permite lanzar o avanzar hacia el cesto, sin oposición. Esta puede tener diferentes dimensiones. Así las categorías de esta variables fueron:

* Medio eficaz por obtención de posesión: cuando tras la realización de medio, el beneficiario principal o secundario obtienen la posesión de balón, aunque no obtengan otra ventaja.

* Medio eficaz por ventaja posicional relativa: se define como aquella ventaja generada gracias a la posición de cualquier oponente perteneciente al medio más cercano al jugador con balón, cuando éste se encuentra con parte de su cuerpo fuera de la línea que une el jugador con balón con el aro.

* Medio eficaz por ventaja posicional máxima. igual que la anterior, a diferencia que en este caso el defensor no está en esa línea imaginaria.

* Medio eficaz por ventaja por distancia: es aquella que se produce cuando existe una distancia entre defensor y jugador con balón suficiente para que éste último pueda lanzar sin oposición. Esta distancia será mayor a un brazo del defensor más cercano (esta distancia no es relativa; lo que varia entre un jugador y otro es el tiempo que necesita desde que recibe hasta que lanza. Por eso se diferenciará si existe o no ventaja cuando recibe, y cuando tira).

* Medio eficaz por ventaja por orientación: se consigue cuando el defensor no está orientado hacia el jugador con balón. Dependerá si la cara anterior del cuerpo, que queda definida por la posición de los pies, está o no orientada al jugador con balón.

* Medio eficaz por ventaja por cercanía al cesto: cuando, sin tener ningún tipo de ventaja anterior, el atacante, por sus características antropométricas y/o físicas, es capaz de lanzar eficazmente dentro del área restringida.

* Medio eficaz por ventaja absoluta: cuando se produzca en combinación al menos dos de las anteriores ventajas, se considerará como absoluta.

* Medio eficaz por enlace eficaz: cuando no exista ninguna de las ventajas anteriores pero los jugadores pertenecientes al medio hayan participado en el enlace con otro, ya sea simultanea o sucesivamente.

La variable independiente estuvo formada por el programa de entrenamiento, el cual se basó en la mejora de cada uno de los Medios Tácticos Colectios Básicos $\left(\mathrm{MTCB}_{\mathrm{S}}\right)$, y en la relación que se establece entre unos y otros. Su duración fue igual al periodo precompetitivo y 
competitivo del equipo con el que se desarrolló el programa, es decir, siete meses.

Los $\mathrm{MTCB}_{\mathrm{S}}$ son las herramientas necesarias para que los jugadores puedan cumplir con los objetivos que marca la lógica del juego, por lo tanto su aprendizaje debe proporcionar las bases conceptuales, procedimentales y actitudinales que permitan su utilización adecuada, atendiendo al cumplimiento de los objetivos del juego. Para ello, se dividió el desarrollo de cada medio en cuatro fases. Para la elección de los objetivos que se iban a trabajar en cada fase se tomaron como criterios: su especificidad, complejidad evolucionando de menor a mayor.

En la primera fase, llamada introductoria a los jugadores se les enseñó, a través de los MTCBs, cuáles son los objetivos del juego: conservar el balón, proteger el cesto, buscar profundad, y lanzar con la menor oposición posible; así como los principios más generales que surgen de ellos: ayuda mutua, seguridad máxima relativa, dificultad la acción defensiva y máxima profundización.

En la segunda fase, o fase de desarrollo, se trabajó el principio específico fundamental de mantener la ventaja obtenida desde un punto de vista colectivo. Para llevar a cabo este principio se requiere de una mayor complejidad, y la necesidad de tener mayor número de herramientas para su realización, puesto que no basta el medio con el que se está trabajando para ponerlo en práctica, sino que habrá que enlazarlo con los que se hayan trabajado anteriormente.

En la fase de perfeccionamiento, una vez que se conoce como utilizar el medio según cada uno de los principios que le afectan, se perfeccionó atendiendo a la actuación de los defensores, según los criterios de eficacia previamente establecidos. Además en esta fase también se desarrolló el principio de aprovechamiento de las ventajas posicionales conseguidas, utilizando para ello el juego en triángulo.

En la cuarta y última fase, la de refuerzo, el jugador se sometió a un refuerzo de lo aprendido hasta ese momento, con dos objetivos: el primero aprender el principio de generar incertidumbre en el rival para conseguir ventajas durante el desarrollo de los medios; y el segundo ser capaz de decidir correctamente en unas condiciones de fatiga tras practicar los medios en unas exigencias físicas mayores.

El instrumento de observación utilizado fue el sistema de categorías, considerado por Anguera (1993) como el instrumento básico de medida en la investigación observacional. Para la validación del instrumento se les pidió a un grupo de expertos formados por doctores en Educación Física y especialistas en baloncesto, que analizarán las categorías de observación. Los observadores que participaron en el estudio fueron alumnos de la asignatura de alto rendimiento en baloncesto de la facultad de ciencias de la actividad física y el deporte de la Universidad de Granada, los cuales recibieron un proceso de adiestramiento según los pasos de Behar (1993). Finalizado éste se realizó el estudio de confiabilidad consiguiendo ser superior al 0,95 .

\subsection{Material y análisis estadístico}

Para la realización de este estudio se utilizó el siguiente material:

* Para el procedimiento de la grabación de los encuentros: una videocámara.

* Para la toma de datos: se diseñó una hoja de observación para la toma de datos de las conductas relacionadas con la eficacia de los ataques y de los MTCB

Los procedimientos estadísticos empleados han sido los que siguen (Perea-Milla, 1998).

\section{Descripción de los datos.}

Mediante la presentación de los resultados en Tablas de Frecuencias proporcionando, junto con los distintos valores o modalidades de cada variable analizada, sus frecuencias absolutas, frecuencias relativas, frecuencias relativas acumuladas y porcentajes de cada modalidad con respecto al tamaño total de la muestra.

\section{Inferencia Estadística.}

El procedimiento empleado ha sido el de Tablas de Contingencia. A través del Test de Chi-Cuadrado se obtuvo tanto la significación unilateral como bilateral que se produce en el cruce de variables.

\section{Resultados}

3.1 Resultados relacionados con la eficacia de los ataques posicionales

Las diferencias entre el pretest y el postest en relación con la eficacia de los ataques posicionales fue muy significativa según el test de Chi-cuadrado, con un $\mathrm{p}=, 006$ (Tabla 1). En el pretest, hubieron 14 ataques en los que se consiguió eficacia, es decir un $28,6 \%$ del total de los ataques posicionales. Mientras que en el último partido de la temporada el $67,5 \%$ de los ataques posicionales fueron eficaces.

\begin{tabular}{|c|c|c|c|c|}
\hline & & \multicolumn{3}{|c|}{ Eficacia ataque Posic. } \\
\hline & & - & + & Total \\
\hline \multirow{4}{*}{ Pretest } & Recuento & 35 & 14 & 49 \\
\hline & $\%$ de Partido & $71,4 \%$ & $28,6 \%$ & $100,0 \%$ \\
\hline & $\%$ de Eficacia ataque & $68,6 \%$ & $36,8 \%$ & $55,1 \%$ \\
\hline & Residuos corregidos & 3,0 & $-3,0$ & \\
\hline \multirow{4}{*}{ Postest } & Recuento & 13 & 27 & 40 \\
\hline & $\%$ de Partido & $4,8 \%$ & $11,6 \%$ & $7,9 \%$ \\
\hline & $\%$ de Eficacia ataque & $32,5 \%$ & $67,5 \%$ & $100,0 \%$ \\
\hline & Residuos corregidos & & & \\
\hline \multirow[t]{3}{*}{ Total } & Recuento & 51 & 38 & 89 \\
\hline & $\%$ de Partido & $57,3 \%$ & $42,7 \%$ & $100,0 \%$ \\
\hline & $\%$ de Eficacia ataque & $100,0 \%$ & $100,0 \%$ & $100,0 \%$ \\
\hline \multicolumn{5}{|c|}{$\begin{array}{l}\text { Corrección por continuidad de la Prueba Chi-cuadrado. Significación: ,006 } \\
\text { Calculado sólo para una tabla de } 2 \times 2 \text {. Estimación de Riesgo de: 3,750 }\end{array}$} \\
\hline
\end{tabular}

También se calculó la estimación de riesgo entre los dos partidos, teniendo la razón de las ventajas un valor de 3,750. Esto quiere decir que la razón de ataques no eficaces con respecto a los ataques eficaces fue 3,750 veces mayor en el pretest con el postest. Estos resultados mostraron que el programa de intervención provocó una mejora en la eficacia del juego con respecto al ataque posicional.

Si se comparan los resultados de la eficacia de los ataques posicionales obtenidos en los partidos seleccionados durante la temporada, se comprueba que, a medida que pasó el tiempo, y por lo tanto a medida que avanzaba el programa de intervención, la eficacia del ataque fue en aumento, mientras que los ataques ineficaces descendían. Hasta el partido 5 , correspondiente a la jornada 14 , los ataques ineficaces superaron a los eficaces. A partir de ese momento el número de ataques eficaces fue siempre superior a los no eficaces, llegando en el partido 8 (jornada 22) a encontrarse las mayores diferencias, con un porcentaje de eficacia del $78,3 \%$ (Figura 1 ).

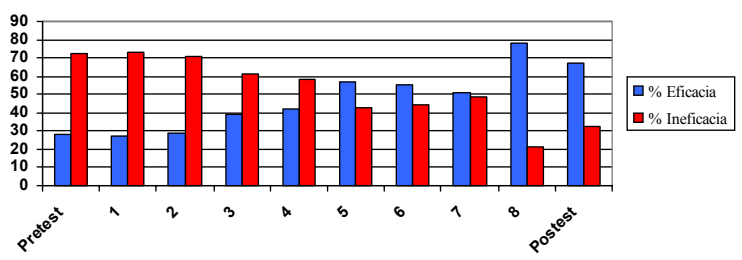

Figura 1. Porcentajes de eficacia de los ataques posicionales durante la temporada

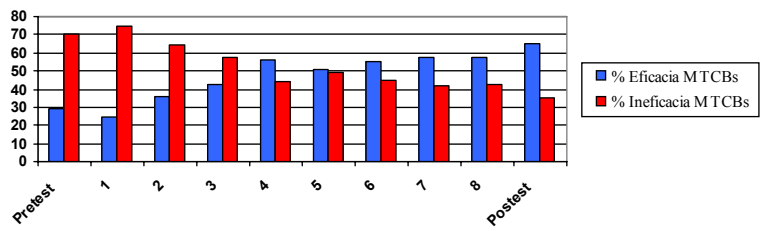

Figura 2. Resultados encontrados sobre la eficacia de los MTCBs durante la temporada.

3.2 Resultados relacionados con la eficacia de los Medios Tácticos Colectivos Básicos

Como se puede comprobar en la tabla 2 , las diferencias entre el pretest y el postest fueron altamente significativas, pasando la eficacia 
del medio de un $29,5 \%$ en el pretest a un $64,9 \%$ en el postest. El grado de mejora entre los dos partidos fue alto, siendo del $35,4 \%$. Esto se refleja en la estimación de riesgo que, en este caso, fue de 6,224, es decir, la razón entre los medios ineficaces con respecto a los eficaces fue 6,224 veces mayor en el pretest que en el postest.

\begin{tabular}{|c|c|c|c|c|}
\hline \multicolumn{2}{|c|}{ Test de evaluación } & \multicolumn{3}{|c|}{ Eficacia del Medio } \\
\hline & & - & + & Total \\
\hline \multirow{4}{*}{ Pretest } & Recuento & 242 & 101 & 343 \\
\hline & $\%$ de partido & $70,5 \%$ & $29,5 \%$ & $100,0 \%$ \\
\hline & $\%$ de Efic. del Medio & $70,3 \%$ & $34,9 \%$ & $54,1 \%$ \\
\hline & Residuos corregidos & 10,5 & $-10,5$ & \\
\hline \multirow{4}{*}{ Postest } & Recuento & 102 & 189 & 291 \\
\hline & $\%$ del partido & $35,1 \%$ & $64,9 \%$ & $100,0 \%$ \\
\hline & $\%$ de Efic. del Medio & $29,7 \%$ & $65,1 \%$ & $45,9 \%$ \\
\hline & Residuos corregidos & $-10,5$ & 10,5 & \\
\hline \multirow[t]{3}{*}{ Total } & Recuento & 344 & 290 & 634 \\
\hline & $\%$ del partido & $35,8 \%$ & $64,2 \%$ & $100,0 \%$ \\
\hline & $\%$ de Efic. del Medio & $100,0 \%$ & $100,0 \%$ & $100,0 \%$ \\
\hline
\end{tabular}

Prueba Chi-cuadrado. Corrección por continuidad $\mathrm{p}=, 000$. Calculado solo para una tabla $2 \times 2$. Estimación de riesgo $=6,224$

Si se comparan los resultados encontrados en los partidos seleccionados durante toda la temporada (Figura 2) se puede comprobar cómo, al igual que la eficacia de los ataques posicionales, la eficacia de los MTCBs creció progresivamente a medida que avanzó la temporada, llegando esta vez su pico al último partido analizado. No es hasta el partido 5 (jornada 14) cuando el número de medios eficaces superó a los no eficaces.

En la figura 3 se puede analizar cómo influyó el programa de intervención en el tipo de eficacia de los MTCBs. Tras la prueba de Chicuadrado de Pearson, las diferencias de los tipos de eficacia entre el pretest y postest fueron altamente significativas $(p=, 000)$, aunque no en todos los tipos las diferencias fueron favorables al postest. La eficacia del medio lograda por la obtención de la posesión fue mucho mayor en el pretest $(41,7 \%)$ que el postest (17,5\%). Esto también ocurrió con la eficacia conseguida por ventaja posicional del jugador con balón, aunque las diferencias fueron bastantes inferiores, siendo de un $9 \%$ en el pretest y de un $3,4 \%$ en el postet. Con respecto a la eficacia conseguida tras una ventaja por distancia del jugador con balón, fue en el postest donde existió un porcentaje mayor $(5,2 \%)$ que en el pretest $(1,5 \%)$, aunque las diferencias, igual que en el caso anterior, fueron mínimas. En la ventaja por cercanía al cesto los resultados fueron prácticamente iguales $(1,5 \%$ y $1 \%$ ) y muy bajos. En el que existieron unas diferencias mayores fue en la ventaja absoluta, encontrándose un $11,3 \%$ en postest, en contraposición de un $3 \%$ en el pretest. Pero, sin duda alguna, fue en la ventaja conseguida por enlace eficaz donde se encontraron las mayores diferencias, puesto que en el pretest no se consiguió ninguna, mientras que en postest el porcentaje se elevó al 26,4\%.

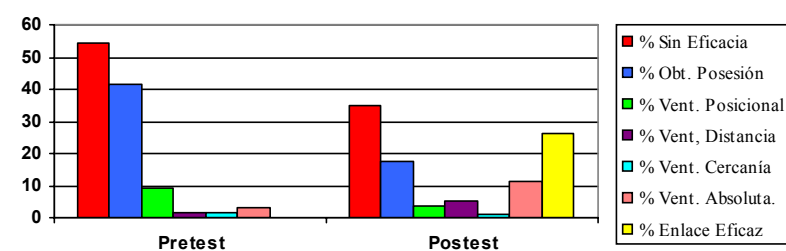

Figura 3. Incidencia del programa de intervención sobre los tipos de eficacia de los MTCBs

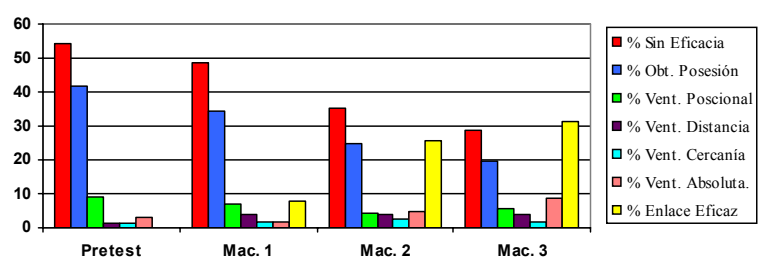

Figura 4. Resultados del tipo de eficacia de los MTCBs en los distintos macrociclos de la temporada
En la figura 4 se pueden observar los tipos de eficacia de los MTCBs que se dieron en los partidos seleccionados de los diferentes macrociclos de los que constaba la planificación anual. El macrociclo 1 está compuesto por los resultados de los partidos 1,2 y 3 (Jornadas 3,7 y 9); el macrociclo 2 por los partidos 4, 5 y 6 (Jornadas 12, 14 y 15 ); y el macrociclo 3 por los partidos 7, 8 y 9 (Jornada 21, 22 y 27).

Los porcentajes de la categoría «sin eficacia» de los medios, realizaron una curva descendente a medida que trascurrió el tiempo, pasando del 54,2\% en el pretest, al 28,7\% en el macrociclo 3 (Figura 4). Lomismo ocurrió con la ventaja conseguida por obtención de la posesión, que pasó del $41,7 \%$ en el pretest al 19,7\% en el macrociclo 3. En cambio, tanto con la ventaja absoluta (del 3\% al 8,6\%) como con la ventaja conseguida por enlace eficaz (del $0 \%$ al 31,5\%) a medida que trascurrió el tiempo tuvieron un crecimiento ascendente. Del resto de tipo de ventajas no pareció encontrarse una constante a lo largo del tiempo.

\section{Discusión}

El objetivo de este estudio fue comprobar si el programa de intervención para la mejora de la táctica colectiva mediante un modelo de enseñanza constructivista mejoraba la eficacia de un equipo en competición. En primer lugar decir que al evaluar la eficacia de los ataques posicionales con la puntuación conseguida en éstos (además de las faltas recibidas), pueden existir algunas variables contaminantes que interfieran en la posible relación que se pueda establecer entre el programa de intervención y la eficacia del juego, como son aquellas relacionadas con la mejora de contenidos de carácter individual. Durante el programa de intervención no se realizó ningún entrenamiento sobre los contenidos individuales como el lanzamiento o el bote, ni desde el punto de vista perceptivo ni en relación con la capacidad para la toma de decisiones, ni de ejecución, fuera de lo establecido por la planificación. Quiere decir esto que el tiempo dedicado al contenido del lanzamiento o el bote estaba relacionado con la mejora de los MTCBs. Por lo tanto, se puede decir que la mejora de la eficacia de los ataques posicionales, con diferencias muy significativas $(\mathrm{p}=, 003)$, tiene una relación directa con el programa de intervención.

Los resultados sobre la eficacia de los ataques posicionales demuestran que para conseguir eficacia en el juego no es necesario invertir el tiempo que proponen los modelos tradicionales a la ejecución, y que la mejora necesaria para ser eficaz se realizará durante tareas globales o analíticas en condiciones más o menos tácticas. Por lo tanto para la eficacia del juego es más importante incidir en la mejora de la toma de decisiones y en el conocimiento conceptual que en la capacidad de ejecución.

Estos resultados no pueden ser comparados con otros anteriores puesto que en ninguno de los estudios analizados en los antecedentes se utiliza como variable dependiente la mejora del rendimiento del juego. Esto puede ocurrir así porque la mayoría de éstos, tanto los relacionados con un modelo constructivista (García López 2004; Harvey et al. 2006; Tallir et al. 2005, 2007) como los relacionados con otros modelos alternativos (Fernández, 2003; Griffin y Placek, 2001; Méndez, 1999; López Parralo, 2004; Turner y Martinek, 1999; Vegas, 2006) están realizados dentro de un marco educativo, ya sea en educación primaria o secundaria, en el cual el rendimiento durante la competición carece de importancia. En aquellos estudios que están realizados en un marco más deportivo como son los de Fabio y Blandón (2003), García Herrero (2001), García y Ruiz (2003), Harvey (2003), Iglesias (2006) o Ponce (2006), creemos que hubiera sido interesante analizar la relación entre sus resultados y la eficacia durante la competición. Sólo en la tesis realizada por Iglesias (2006) se analiza el porcentaje de acierto en el lanzamiento durante la competición de manera aislada y con el objetivo de evaluar la ejecución del lanzamiento. Los resultados mostraron que con el programa de intervención basado en la reflexión estos porcentajes también mejoraron, pudiéndose deber este hecho a que «lanzar en situaciones tácticas propicias para ello, también suponga mayores probabilidades de acierto en la ejecución, aunque esta cuestión debe ser 
interpretada con cautela» (Iglesias, 2006, p. 136). Aun así, este dato no es suficiente para conocer si la eficacia del juego pudo aumentar por el programa de reflexión realizado, puesto que no se sabe si se consiguieron más o menos lanzamientos, o más o menos faltas recibidas.

Si se analizan las categorías de la variable eficacia de los MTCBs existe un dato que puede llevar a confusión. En el caso de la eficacia conseguida por ventaja posicional, los resultados del pretest son superiores a los del postest. Esto se debe a que en el postest se consiguió un mayor número de eficacias por ventaja absoluta. Es decir, en el test final se contabilizan muy pocas ventajas posicionales, no porque se dieran menos que en el pretest, sino porque éstas iban acompañadas además de otro tipo de ventaja, como la conseguida por distancia o por orientación, por lo que éstas se anotaban como ventaja absoluta.

Los valores de eficacia de los MTCBs son muy similares a los conseguidos por la eficacia de los ataques posicionales, es decir que a medida que aumenta la eficacia de los primeros, también lo hace la eficacia de los segundos, con porcentajes prácticamente iguales en el postest $(65,1 \%$ para la eficacia de los medios y $67,5 \%$ para la eficacia de los ataques posicionales). Si se relacionan ambas variables, las diferencias encontradas tras la corrección por continuidad de la prueba de Chicuadrado para una tabla $2 \times 2$, son altamente significativas $(p=, 000)$. Quiere decir que conseguir eficacia en los MTCBs influye positivamente en el rendimiento de los ataques posicionales.

\section{Conclusiones}

La principal conclusión del estudio es que un programa de intervención para la mejora de la táctica colectiva, que utiliza un modelo de enseñanza constructivista basado en la reflexión y la provocación de conflictos ha demostrado ser eficaz para aumentar el rendimiento del equipo seleccionado, al aumentar la eficacia de los ataques posicionales y la eficacia de los MTCBs durante el juego real.

Además, conseguir mejoras en la eficacia de los MTCBs, es decir, conseguirunas mejores condiciones de lanzamiento gracias a unas ventajas espaciales influye directamente en una mejora de los ataques posicionales, es decir, en conseguiréxito.

\section{Referencias bibliografías}

Amorim,J.(2001). Relaçao entre as variáveis técnico-tácticas e clssificaçao final: um estudo na Liga Portuguesa de Basquetebol.EnF. J. Tavares, M. A Graça, D. Pinto y E. Brandao (Ed.), Tendências actuais da investigaçao em Basquetebol. (pp. 80-89). Oporto.: Facultade de Ciências do Desporto e de Educaçao Física da Universidad do Porto.

Angera, M. T. (1993). Metodología observacional en la investigación psicológica. Barcelona: PPU, S.A.

Alarcón, F. (2008). Incidencia de un programa de entrenamiento para la mejora de la táctica colectiva del ataque posicional de un equipo de baloncesto masculino. Tesis doctoral no publicada. Granada: Universidad de Granada.

Behar, J. (1993). Sesgos del observador. En M.T. Anguera (Ed.), Metodología observacional en la investigación Psicológica. (pp. 27-76). Barcelona: PPU.

Buendía, L., Colás, P. y Hernández, F. (1998). Métodos de investigación en Psicopedagogía. Madrid: McGraw-Hill.

Butler, J. I. ( 1996). Teacher responses to teaching games for understanding. Teachers are interviewed about the attractions and drawbacks of the tactical approach. The Journal of physical education, recreation and dance, 67 (9), 17-20.

Cárdenas, D. (1999). Proyecto docente: asignatura. Fundamentos de los deportes colectivos: Baloncesto. Manuscrito no publicado. Universidad de Granada.

Cárdenas, D. (2005). Fundamentos de las habilidades de los deportes de equipo: baloncesto. Granada: Servicio de Copias de la FCCAF y D.
Contreras, O. (1998). Didáctica de la Educación Física. Un enfoque constructivista. Barcelona: Inde.

Fabio, H. y Blandón, M. (2003). Enseñanza proposicional para la formación de la táctica en el fútbol. Lecturas: Educación Física y Deportes, 65. Extraído el 12 de febrero del 2004 desde http:// www.efdeportes.com/efd65/futbol.htm.

Fernández,F. (2003). La enseñanza del deporte con diferentes programas: técnico, táctico, técnico-táctico. II Congreso Ibérico de baloncesto. Propuestas para la mejora en el proceso de formación y en el rendimiento en baloncesto. Cáceres, 27,28 y 29 de Noviembre.

Fierro, C. (2002). Variables relacionadas con el éxito deportivo en las ligas NBAy ACB de baloncesto. Revista de psicología del deporte, 11(2), 247-255.

García Herrero, J. A. (2001). Adquisición de la competencia para el deporte en la infancia. El papel del conocimiento y la comprensión en la toma de decisiones en balonmano. Tesis Doctoral no publicada. Extremadura: Universidad de Extremadura.

García Herrero, J.A. y Ruiz, L. M. (2003). Análisis comparativo de dos modelos de intervención en el aprendizaje del balonmano. Revista de psicología del Deporte, 12 (1), 55-66.

García López, L. M. (2004). Transferencia en los modelos horizontales de iniciación deportiva. Tesis Doctoral no publicada. Castilla la Mancha: Universidad de Castilla la Mancha.

Gómez, M. A. Y Lorenzo, A. (2005). Diferencias entre equipos ganadores y perdedores en el rendimiento de competición en baloncesto femenino. Kronos, 8, (4) 16-19.

González, S., Gutiérrez, D., Pastor, J. y Fernández J. (2007). El proceso de enseñanza-aprendizaje del Baloncesto en las escuelas deportivas. Propuesta de programación para la categoría Benjamín. Retos: nuevas tendencias en educación física, deporte y recreación, $\mathrm{n}^{\circ} 11$, 17-25.

Grehaigne, J. F., Godbout, P. \& Bouthier, D. (1999). The foundations of tactics and strategy in team sports. Journal of Teaching in Physical Education, 18 (2), 159-174.

Grehaigne, J. F., Godbout, P. \& Bouthier, D. (2001). The teaching and learning of decision making in team sports. Quest, 53 (1), 59-76.

Griffin, L.\& Placek, J. L. (2001). The Understanding and development of learners' Domain-Specific Knowledge. Journal of teaching in physical education, 20 (4), 299-407.

Harvey, S. (2003). Teaching Games for Understanding: A study of U19 college soccer players improvement in game performance using the Game Performance Assessment Instrument. 2nd International Conference: Teaching Sport and physical Education for understanding University of Melbourne. Australia.

Harvey, S., Bryan, R., Weigs, H., González, A. \& Van Der Mars, H. (2006). Effects of Teaching Games for Understanding on Game Performance and Understanding in Middle School Physical Education. Physical education \& sport pedagogy, 3, 74-168.

Iglesias, D. (2006). Efecto de un protocolo de supervisión reflexiva sobre el conocimiento procedimental, la toma de decisiones y la ejecución en jugadores jóvenes de baloncesto. Tesis Doctoral no publicada. Extremadura: Universidad de Extremadura.

Karipidis, A., Fotinakis, P., Taxildaris, K. , Filaktakidou, A. \& Fatouros, J. (2001). Factors characterisingva succesful performance in basketball. Journal of human movement studies, 41(5), 385-397.

Latorre, A., Del Rincón, D. y Arnal, J. (2003). Bases metodológicas de la investigación educativa. Barcelona: Experiencia.

López Parralo, M. J. (2004). Análisis comparativo de dos metodologías en iniciación deportiva a través del diario del profesor. Lecturas: Educación Física y Deportes 10 (79). Extraído el 3 de Noviembre del 2005 de http://www.efdeportes.com/efd79/diario.htm.

Manhao, C.,A. (2000). A importância dos indicadores técnico-tácticos na discriminaçao da performance em Basquetebol: um estudo na liga profissional norte americana. Unpublished Monografia de Licenciatura., ESEFD-IPM. 
Mcmorris, T. \& Graydon, J. (1997). The contribution of the research literature to the undestanding of decision making in team games. Journal of human movement studies, 33, 69-90.

Méndez,A. (1999). Análisis comparativo de dos técnicas de enseñanza en la iniciación a dos deportes de invasión: el floorball patines y el baloncesto. Tesis Doctoral no publicada. Granada: Universidad de Granada.

Musch, E., Mertens, B., Graça,A., Timmers, E., Meertens, T., Taborsky, F. et al. (2002). An innovated didactical invasion games model to teach basketball and handball, presented on CD. 7th Congress of European College of Sport Science. Atenas.

Nuviala,A. y Tamayo, J. (2003). Las tareas motrices en la enseñanza de los elementos técnico-tácticos en los deportes. Retos: nuevas tendencias en educación fisica, deporte y recreación, 4, 5-10.

Perea-Milla, E. (1998). Estudios cuasi-experimentales y estudios aleatorios controlados. En R. Burgos (Eds), Metodología de investigación y escritura científica en clínica (pp.147-176). Granada: Escuela Andaluza de Salud Pública.

Piñar, M. I. (2005). Incidencias del cambio de un conjunto de reglas de juego sobre algunas variables que determinan el proceso de aprendizaje de los jugadores de minibasket. Tesis Doctoral no publicada. Granada: Universidad de Granada.

Ponce, F. (2006). La eficacia de una técnica de enseñanza mediante la búsqueda o indagación en la mejora de diferentes aspectos funcionales, técnico-tácticos, decisionales y motivacionales, en futbolistas de categoría alevin, frente a una intervención tradicional y su transferencia a la competición. Tesis Doctoral no publicada. Granada: Universidad de Granada.

Refoyo, I. (2001). La decisión táctica de juego y su relación con la respuesta biológica de los jugadores. Una aplicación al baloncesto como deporte de equipo. Tesis Doctoral no publicada. Madrid: Universidad Politécnica de Madrid.

Sampaio,A. (2000). Opoder Discriminatorio das Estatísticas Do Jogo De Basquetebol Em Diferentes Contextos. Novos Caminos Metodológicos De Análise. Vila Real: Unpublished Dissertaçao De Doctouramento.

Sampaio, J. (1998). Los indicadores estadísticos más determinantes en el resultado de los partidos de básquetbol. Lecturas: educación fisica y deportes, Revista Digital, 11. Extraído el 3 de Noviembre del 2005 de http://www.efdeportes.com/efd11/sampe.htm.

Sampaio, J. y Leite, N. (2006). ¿Por qué ganaron o perdieron los partidos de baloncesto los equipos que participaron el Eurobasket 2005. Kronos, 9, (5) 67-73.
Satter, D. J. (2003). An empirical analysis of the final four participants in the NCAA Division Imen's basketball tournament, 1987-2003. Tesis no publicada. University of North Carolina, Chapel Hill.

Sierra-Bravo, R. (1996). Técnicas de investigación social. Madrid: Paraninfo.

Tallir, I. M. E., Lenoir, M. Valcke, M. \& Musch, E. (2005). Effects of Two Instructional Approaches for Basketball on Decision-making and Recognition Ability. International journal of sport psychology, 36 (2), 107-126.

Tallir,I. M.E., Lenoir, M., Valcke, M. \& Musch,E.(2007). Do alternative instructional approaches result in different game performance learning outcomes? Authentic assessment in varying game conditions. International journal of sport psychology, 38(3), 263282.

Tsamourtzis, E., Fylaktakidou, A, \& Taxildaris, K. (2001). The role that the time of the offensive duration plays to the efficacy of a basketball team. Exercise \& society journal of sports science, 27, $32-43$.

Tsamourtzis, E. \& Athanasiou, N. (2004). Registration of rebound possession zones in basketball. International journal of performance analysis in sport, 4(1), 34-39.

Turner, A. P. \& Martinek, T. J. (1999). An investigation into teaching games for understanding: effects on skill, knowledge, and game play. Research quarterly for exercise and sport, 70 (3), 286-296.

Ureña, N., Alarcón, F. y Ureña, F. (2009). La realidad de los deportes de equipo en la enseñanza secundaria. Cómo planifican e intervienen los profesores de Murcia. Retos: nuevas tendencias en educación fisica, deporte y recreación, $\mathrm{n}^{\circ} 16,9-15$.

Vegas, G. (2006). Metodología de enseñanza basada en la implicación cognitiva del jugador de fútbol base. Tesis Doctoral no publicada. Granada: Universidad de Granada.

Vidal, J., Y Mercadé, J. J. (2002). Incidencia de la entrada a canasta en el resultado final de un encuentro de baloncesto. Un caso práctico de análisis observacional. In M. Zabala, Chirosa, I, Chirosa, L, y Viciana,J.(Ed.), Tecnología cientifica aplicada al controly evaluación del Rendimiento Deportivo. (pp. 353-369). Granada: Facultad de Ciencias de la Actividad Física y del Deporte.

Wright, S., Mcneill, M., Fry, J. \& Wang, J. (2005). Teaching teachers to play and teach games. Physical education \& sport Pedagogy, 10 (1), 61-82.

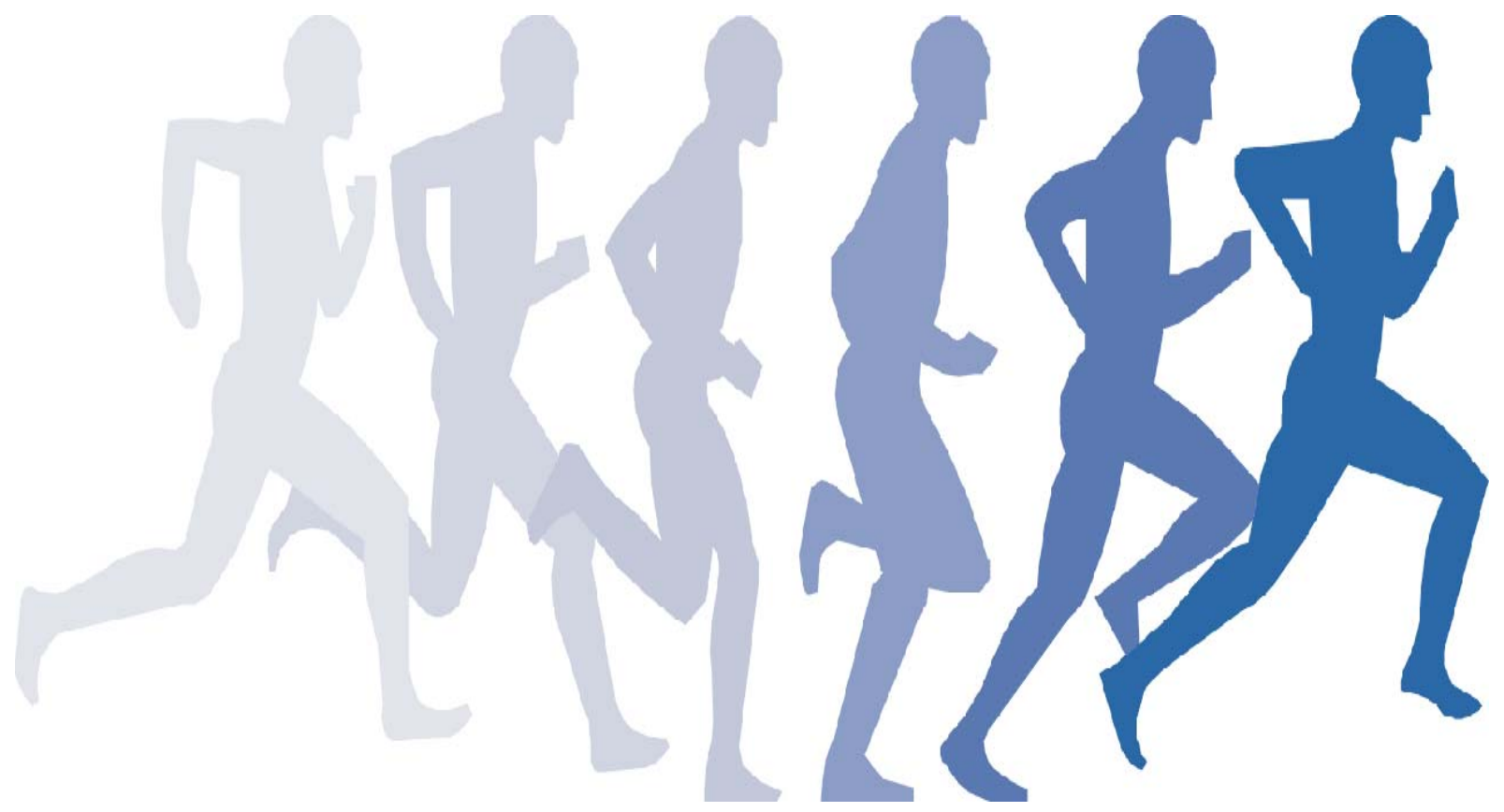

